\title{
Association of HLA genotypes with Beta-lactam antibiotic hypersensitivity in children
}

\author{
Prapasiri Singvijarn, ${ }^{1}$ Wiparat Manuyakorn, ${ }^{1}$ Surakameth Mahasirimongkol, ${ }^{2}$ Sukanya Wattanapokayakit, ${ }^{2}$ Wimala Inunchot, ${ }^{2}$ \\ Nuanjun Wichukchinda, ${ }^{2}$ Supharat Suvichapanich, ${ }^{3}$ Wasu Kamchaisatian, ${ }^{1}$ Suwat Benjaponpitak ${ }^{1}$
}

\begin{abstract}
Background: Beta-lactam (BL) antibiotics hypersensitivity is common in children. Clinical manifestation of BL hypersensitivity varies from mild to severe cutaneous adverse drug reactions (SCARs).
\end{abstract}

Objective: To determine the association of HLA genotype and BL hypersensitivity and the prevalence of true drug allergy in patients with history of BL hypersensitivity.

Methods: A case-control study was performed in 117 children with aged 1-18 years. Children with history of nonSCARs BL hypersensitivity were evaluated for true drug hypersensitivity including skin test and drug provocation test. Tolerant control patients were children who could tolerate BL for at least 7 days without hypersensitivity reaction. HLA genotype (HLA-A, HLA-B, HLA-C and HLA-DRB1) were performed in 24 cases and 93 tolerant controls using PCRSSO (polymerase chain reaction - sequence specific oligonucleotide probes).

Results: There were association of $H L A-C^{*} 04: 06$ (OR $=13.14,95 \% C I: 1.3-137.71 ; p=0.027$ ), and HLA-C*08:01 (OR $=4.83,95 \%$ CI: $1.93-16.70 ; p=0.016)$ with $\mathrm{BL}$ hypersensitivity. $H L A-B^{\star} 48: 01$ was strongly associated with immediate reaction from $\mathrm{BL}$ hypersensitivity $(\mathrm{OR}=37.4,95 \% \mathrm{CI}$ : $1.69-824.59 ; p=0.016)$ while $H L A-C^{\star} 04: 06, H L A-C^{\star} 08: 01$ and $H L A-D R B 1^{*} 04: 06$ were associated with delayed reaction $(p<0.05)$. Among 71 cases who were newly evaluated for BL hypersensitivity, only 7 cases $(9.8 \%)$ had true BL hypersensitivity.

Conclusions: Less than $10 \%$ of children with suspected of BL hypersensitivity have true hypersensitivity. There might be a role of HLA-B, HLA-C and HLA-DRB1 genotype in predicting BL hypersensitivity in Thai children.

Key words: $H L A-B^{\star} 48: 01, H L A-C^{\star} 04: 06, H L A-C^{\star} 08: 01, H L A-D R B 1^{\star} 04: 06$, Drug reaction, Thai

\footnotetext{
Affiliations:

Department of Pediatrics, Faculty of Medicine Ramathibodi Hospital, Mahidol University, Bangkok, Thailand

${ }^{2}$ Medical Genetics Center, Medical Life Sciences Institute, Department of Medical Sciences, Ministry of Public Health, Nonthaburi, Thailand Department of Biochemistry, Faculty of Pharmacy,

Mahidol University, Bangkok, Thailand
}

\section{Introduction}

Beta-lactam (BL) antibiotics including penicillin and cephalosporin antibiotics are commonly used for bacterial infection in children. Approximately $10-20 \%$ of children receiving beta-lactam antibiotics develop hypersensitivity reaction. ${ }^{1-3}$ Drug hypersensitivity reactions can be classified simply as immediate or delayed reaction. Immediate reactions occur within 1 hour after intake of the drug and are often mediated by IgE antibodies. ${ }^{4}$ Clinical symptom of immediate reaction can be local organ involvement such as urticaria, angioedema,

\section{Corresponding author:} Wiparat Manuyakorn Division of Allergy and Immunology, Department of Pediatrics, Faculty of Medicine Ramathibodi Hospital, Mahidol University Rama 6 Rd, Ratchathewi, Bangkok 10400, Thailand

E-mail: mwiparat@hotmail.com bronchospasm, gastrointestinal symptoms, and multi-organ involvement as anaphylaxis. Non-immediate or delayed reactions occur later than 1 hour. Its clinical can be maculopapular rash, fixed drug eruptions, and severe cutaneous adverse drug eruptions (SCARs) including exfoliative dermatitis, acute generalized exanthematous pustulosis (AGEP), Stevens-Johnson syndrome (SJS), toxic epidermal necrolysis (TEN), and drug reaction with eosinophilia and systemic symptoms (DRESS). ${ }^{5}$ In children, it is difficult to distinguish rashes 
from viral exanthem and drug induced hypersensitivity. As a result, antibiotics hypersensitivity is over-diagnosed and leading to administration of alternative antibiotic with increasing costs and development of drug resistance due to the increased usage of broad spectrum antibiotics. ${ }^{6}$

Recently there is increasing evidence on the role of pharmacogenomics testing in pediatric patients to predict the dose requirement, efficacy and adverse drug reaction. ${ }^{7}$ However, ethnicity also has an impact on the genetic frequency. $H L A-B^{\star}$ 15:02 has been demonstrated to have a strong association with carbamazepine induced SJS/TEN in Han Chinese ${ }^{8}$ and Thais ${ }^{9,10}$ but not in Japanese or European population. ${ }^{11}$ Recommendation to screen for $H L A-B^{\star} 15$ :02 before prescribing carbamazepine $e^{12,13}$ has been used in several Asian countries including Thailand. We have recently demonstrated the role of pharmacogenetics testing in predicting phenytoin hypersensitivity ${ }^{14}$ and phenobarbital hypersensitivity. ${ }^{15,16}$ However, there is limited study investigating the association between HLA genotypes and BL hypersensitivity in children. The present study aimed to determine the association of HLA genotype and beta lactam hypersensitivity in children and demonstrated the prevalence of true drug hypersensitivity in patients with history of BL hypersensitivity.

\section{Methods \\ Subjects}

A case-control study was performed in 117 children at Department of Pediatrics, Faculty of Medicine Ramathibodi Hospital, Mahidol University, Bangkok, Thailand. Cases were Thai children aged 1-18 years with confirmed diagnosis of BL hypersensitivity defined as positive BL hypersensitivity investigation in addition to having history of suspected BL hypersensitivity or having the diagnosis of SCARs from BL. The diagnosis of SCARs were confirmed by either an allergist or a dermatologist, and their decisions were based on the clinical criteria for SJS/TEN ${ }^{17}$ and DRESS. ${ }^{18}$ Tolerant controls were healthy Thai children with no allergic reaction to $\mathrm{BL}$ drug provocation (drug tolerance). This study was reviewed and approved by the human rights and ethic committee of Faculty of Medicine, Ramathibodi Hospital, Mahidol University (ID 01-57-17).

\section{True BL hypersensitivity investigation}

All subjects with suspected BL hypersensitivity without clinical of severe cutaneous adverse drug eruption (SCARs) were evaluated for the confirmation of true BL hypersensitivity as previously reported. ${ }^{2}$ Briefly skin prick test was initially performed followed by intradermal skin test if the result of skin prick test was negative. Drug provocation test (DPT) was performed in cases with negative skin test result.

\section{HLA Genotyping}

After written informed consent was obtained, the genomic DNA was extracted from the peripheral blood and diluted to $20 \mathrm{ng} / \mu \mathrm{L}$, according to the established DNA extraction protocol protocols of the manufacturer. The HLA genotype determination and the data analysis was performed by WAKflow software (Wakunaga, Osaka, JAPAN).
HLA genotyping was based reverse sequence-specific oligonucleotide probes (SSO). The HLA genotyping is typed to 2-fileld (4 digits).

\section{Statistical analysis}

Data analysis was performed using SPSS 18.0 software package and $\mathrm{R}$ console 3.1.1 statistical software for window (http://cran.r-project.org/). Descriptive statistic were used for demographic data. Genotype carrier rate were compared between the drug allergy group and the drug tolerance group. The associations of HLA alleles/genotype carrier rate with BL hypersensitivity were determined by Fisher's exact test, and the strength of association was estimated by calculating the odds ratios (ORs) and 95\% confidence intervals (CIs). Haplotype association analysis were carried out using 'haplo.stats' packages. The test associations were determined from $P$-values. ORs and 95\% CIs were obtained using inferred counts of haplotypes. A $p$-value less than 0.05 was considered statistically significant.

\section{Results}

One hundred and seventeen subjects were enrolled: 71 children with suspected of BL hypersensitivity who were evaluated for true BL hypersensitivity, 15 cases of previously confirmed BL hypersensitivity, 2 cases with the diagnosis of SCARS (1 SJS and 1 DRESS) and 29 children with known BL tolerance. All 71 children who underwent for true BL hypersensitivity had histories of reactions after exposure to $\mathrm{BL}$ as follow: 30 with urticaria/angioedema, 3 with anaphylaxis, 37 with maculopapular eruption and 1 with fixed drug eruption. Only 7 (9.8\%) out of 71 children with suspected of BL hypersensitivity were confirmed to have BL hypersensitivity (Figure 1) A total of 24 children with confirmed BL hypersensitivity (case) and 93 children with BL tolerance (control) were analyzed in the case control study. There was no significant differences in the mean age between case and control $(9.04 \pm$ 4.31 year vs $8.03 \pm 4.12$ year, $p=0.51$ ). Amoxicillin was the most common cause of true BL hypersensitivity in our study (58.3\%), followed by amoxicillin-clavulanic acid (16.7\%), ceftriaxone $(8.3 \%)$, cloxacillin (8.3\%), ampicillin/salbactam (4.2\%) and meropenem (4.2\%). Fourteen (58.3\%) of 24 children had immediate reactions. Urticarial rash appeared in 14 children, two children were diagnosed anaphylaxis, maculopapular eruptions were presented in 5 children and SCARs were diagnosed in 2 cases ( 1 case with DRESS, 1 case with SIS) (Table 1).

\section{Association of HLA genotype Carrier Rates and the BL hy- persensitivity}

The HLA-A, HLA-B, HLA-C and HLA-DRB1 genotypes of BL hypersensitivity are listed in Table 1. There are trends of higher carrier rates of $H L A-B^{\star} 48: 01$ and $H L A$ $D R B 1^{\star} 04: 06$ in the $\mathrm{BL}$ hypersensitivity than those in the BL tolerant control group $(8.3 \%$ vs. $0 \%$, OR 20.78 ; $95 \% \mathrm{CI}$ : $0.96-448.06 ; p=0.053, p c=n s)$. Significant association of $H L A-C^{\star} 04: 06$ and $H L A-C^{\star} 08: 01$ with the BL hypersensitivity was demonstrated. The carrier rates of $H L A-C^{\star} 04$ :06 were $12.5 \%$ and $1.1 \%$ in children who had BL hypersensitivity 


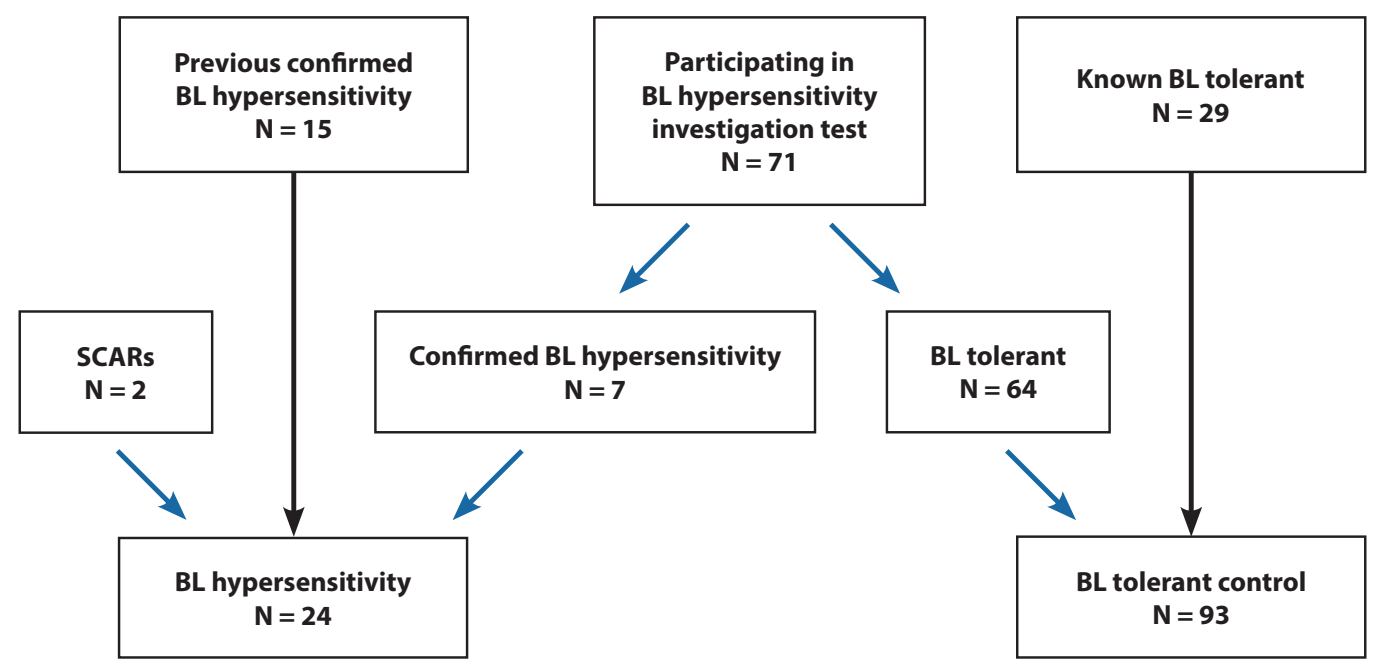

Figure 1. The flow of patient enrollment.

Table 1. HLA class I genotypes of beta-lactam hypersensitivity reaction

\begin{tabular}{|c|c|c|c|c|c|c|c|c|c|c|c|c|}
\hline ID & Drug & Clinical & Type & $\begin{array}{c}\text { Positive } \\
\text { Test }\end{array}$ & \multicolumn{2}{|c|}{ HLA- A } & \multicolumn{2}{|c|}{ HLA- B } & \multicolumn{2}{|c|}{ HLA- C } & \multicolumn{2}{|c|}{ HLA- DRB 1} \\
\hline 1 & cloxacillin & anaphylaxis & I & DPT & $A^{*} 02: 03$ & $A * 29: 01$ & $B^{\star} 18: 01$ & $B * 37: 01$ & $C^{\star} 06: 02$ & $C^{*} 07: 01$ & $D R B 1^{*} 10: 01$ & $D R B 1^{*} 14: 10$ \\
\hline 2 & amoxicillin & anaphylaxis & I & ID & $A^{*} 02: 01$ & $A^{*} 02: 03$ & $B^{\star} 15: 02$ & $B^{\star} 18: 01$ & $C^{*} 07: 04$ & $C^{\star} 08: 01$ & $D R B 1^{*} 12: 02$ & $D R B 1^{*} 15: 02$ \\
\hline 3 & amoxicillin & urticaria & I & DPT & $A^{\star} 11: 01$ & $A^{\star 11: 01}$ & $B^{\star} 13: 01$ & $B * 39: 01$ & $C^{\star} 03: 04$ & $C^{\star 07: 01}$ & $D R B 1^{\star} 04: 03$ & $D R B 1^{*} 15: 01$ \\
\hline 4 & amoxicillin & urticaria & I & DPT & $A^{* 02: 07}$ & $A^{*} 11: 01$ & $B^{\star} 15: 02$ & $B \star 56: 02$ & $C^{*} 01: 02$ & $C^{\star} 08: 01$ & $D R B 1^{*} 14: 01$ & $D R B 1^{*} 15: 02$ \\
\hline 5 & amoxicillin & urticaria & I & SPT & $A^{\star 02: 01}$ & $A^{* 02: 07}$ & $B^{\star} 15: 01$ & $B^{\star} 40: 02$ & $C^{\star} 03: 03$ & $C^{\star} 15: 02$ & $D R B 1^{\star} 04: 05$ & $D R B 1^{*} 16: 02$ \\
\hline 6 & amoxicillin & urticaria & I & ID & $A^{*} 02: 01$ & $A * 26: 01$ & $B^{\star 38: 01}$ & $B \star 52: 20$ & $C^{\star} 07: 01$ & $C^{\star} 12: 02$ & $D R B 1^{*} 14: 04$ & $D R B 1^{*} 15: 01$ \\
\hline 7 & amoxicillin & urticaria & I & ID & $A^{\star} 11: 01$ & $A * 34: 01$ & $B^{\star} 15: 141$ & $B^{\star} 40: 87$ & $C^{\star} 07: 01$ & $C^{\star 07: 02}$ & $D R B 1^{*} 12: 01$ & $D R B 1^{*} 15: 02$ \\
\hline 8 & ceftriaxone & urticaria & I & DPT & $A^{* 02: 07}$ & $A * 24: 07$ & $B^{* 35: 05}$ & $B \star 48: 01$ & $C^{\star} 04: 01$ & $C^{\star} 08: 01$ & $D R B 1^{\star} 04: 05$ & $D R B 1^{*} 07: 01$ \\
\hline 9 & amoxicillin & urticaria & I & SPT & $A^{* 02: 03}$ & $A * 33: 03$ & $B^{\star} 13: 01$ & $B^{\star} 44: 03$ & $C^{\star} 04: 06$ & $C^{\star 07: 01}$ & $D R B 1^{*} 07: 01$ & $D R B 1^{*} 12: 02$ \\
\hline 10 & amoxicillin & urticaria & I & ID & $A^{* 02: 07}$ & $A * 33: 03$ & $B^{\star} 44: 03$ & $B^{\star} 46: 01$ & $C^{\star} 01: 02$ & $C^{*} 07: 01$ & $D R B 1^{*} 07: 01$ & $D R B 1^{*} 12: 02$ \\
\hline 11 & amoxicillin & urticaria & I & ID & $A^{*} 11: 01$ & $A * 24: 02$ & $B^{\star} 15: 01$ & $B^{\star} 46: 01$ & $C^{*} 01: 02$ & $C^{*} 04: 01$ & $D R B 1^{*} 04: 05$ & $D R B 1^{*} 16: 02$ \\
\hline 12 & amoxicillin & urticaria & I & SPT & $A^{\star} 11: 01$ & $A^{\star} 11: 01$ & $B^{\star 27: 06}$ & $B \star 48: 01$ & $C^{\star} 01: 02$ & $C^{\star} 03: 04$ & $D R B 1^{\star} 12: 02$ & $D R B 1^{*} 15: 02$ \\
\hline 13 & amoxicillin & urticaria & I & ID & $A^{*} 11: 02$ & $A * 33: 03$ & $B^{\star} 13: 01$ & $B^{\star} 44: 03$ & $C^{\star} 07: 01$ & $C^{\star} 12: 02$ & $D R B 1^{*} 07: 01$ & $D R B 1^{*} 14: 01$ \\
\hline 14 & cloxacillin & urticaria & I & SPT & $A^{\star} 23: 14$ & $A^{*} 33: 59$ & $B^{\star} 40: 01$ & $B^{\star 58: 01}$ & $C^{\star} 03: 02$ & $C^{\star 03: 04}$ & $D R B 1^{\star} 07: 01$ & $D R B 1^{\star} 11: 01$ \\
\hline 15 & Amoxi/clavu & urticaria & $\mathrm{D}$ & ID & $A^{*} 02: 07$ & $A^{* 11: 01}$ & $B^{\star 38: 02}$ & $B^{\star} 46: 01$ & $C^{\star} 01: 02$ & $C^{*} 07: 01$ & $D R B 1^{*} 11: 01$ & $D R B 1^{*} 16: 02$ \\
\hline 16 & amoxicillin & urticaria & $\mathrm{D}$ & DPT & $A^{*} 02: 07$ & $A^{* 11: 01}$ & $B^{\star 07: 05}$ & $B^{\star} 13: 01$ & $C^{\star} 03: 04$ & $C^{\star 04: 06}$ & $D R B 1^{*} 12: 02$ & $D R B 1^{*} 16: 02$ \\
\hline 17 & Amoxi/clavu & Fix drug & $\mathrm{D}$ & ID & $A^{*} 11: 01$ & $A^{*} 11: 01$ & $B^{\star} 08: 01$ & $B^{\star} 15: 02$ & $C^{\star} 07: 01$ & $C^{\star 08: 01}$ & $D R B 1^{\star} 03: 01$ & $D R B 1^{*} 12: 02$ \\
\hline 18 & Amoxi/clavu & MPE & $\mathrm{D}$ & ID & $A^{*} 11: 01$ & $A * 24: 02$ & $B^{* 27: 06}$ & $B^{\star} 40: 01$ & $C^{\star} 03: 04$ & $C^{\star} 07: 01$ & $D R B 1^{\star} 04: 06$ & $D R B 1^{*} 16: 02$ \\
\hline 19 & ceftriaxone & MPE & $\mathrm{D}$ & ID & $A^{*} 02: 03$ & $A * 33: 03$ & $B^{\star} 13: 01$ & $B^{\star} 44: 03$ & $C^{\star 04: 06}$ & $C^{\star} 07: 01$ & $D R B 1^{*} 07: 01$ & $D R B 1^{*} 16: 02$ \\
\hline 20 & amoxicillin & MPE & $\mathrm{D}$ & DPT & $A^{*} 11: 01$ & $A^{* 11: 01}$ & $B^{*} 35: 03$ & $B^{\star} 40: 01$ & $C^{\star} 03: 03$ & $C^{\star} 04: 01$ & $D R B 1^{\star}{ }^{\star 04: 06}$ & $D R B 1^{*} 12: 02$ \\
\hline 21 & amoxicillin & MPE & $\mathrm{D}$ & DPT & $A^{*} 11: 01$ & $A * 24: 02$ & $B^{\star} 40: 01$ & $B \star 54: 01$ & $C^{\star} 01: 02$ & $C^{\star 07: 01}$ & $D R B 1^{*} 04: 04$ & $D R B 1^{*} 04: 05$ \\
\hline 22 & Amoxi/clavu & MPE & $\mathrm{D}$ & DPT & $A^{\star 01: 01}$ & $A * 24: 07$ & $B^{\star} 13: 01$ & $B^{\star} 15: 02$ & $C^{\star} 07: 01$ & $C^{\star 08: 01}$ & $D R B 1^{*} 12: 02$ & $D R B 1^{*} 15: 02$ \\
\hline 23 & Ampi/sulbac & SJS & $\mathrm{D}$ & ND & $A^{*} 11: 01$ & $A^{\star 2} 24: 02$ & $B^{\star} 18: 01$ & $B^{\star 52: 01}$ & $C^{\star} 05: 01$ & $C^{\star} 12: 02$ & $D R B 1^{\star} 04: 03$ & $D R B 1^{*} 04: 84$ \\
\hline 24 & meropenam & DRESS & $\mathrm{D}$ & ND & $A * 24: 02$ & $A^{*} 74: 01$ & $B^{\star} 15: 02$ & $B^{\star} 15: 02$ & $C^{\star} 08: 01$ & $C^{\star 08: 01}$ & $D R B 1^{*} 12: 02$ & $D R B 1^{*} 14: 22$ \\
\hline
\end{tabular}

amoxi/clavu = amoxicillin-clavulanic acid, ampi/sulbac $=$ ampicillin/salbactam, $\mathrm{I}=$ immediate reaction, $\mathrm{D}=\mathrm{Delayed}$ reaction, $\mathrm{MPE}=\mathrm{maculopapular}$ eruption, SJS $=$ Stevens - Johnson syndrome, DRESS $=$ Drug reaction with eosinophilia and systemic symptoms. SPT $=$ skin prick test, ID-intradermal skin test, DPT $=$ drug provocation test. ND = not done. Alleles with significant higher carrier rate compared with tolerant control are highlighted in bold. 

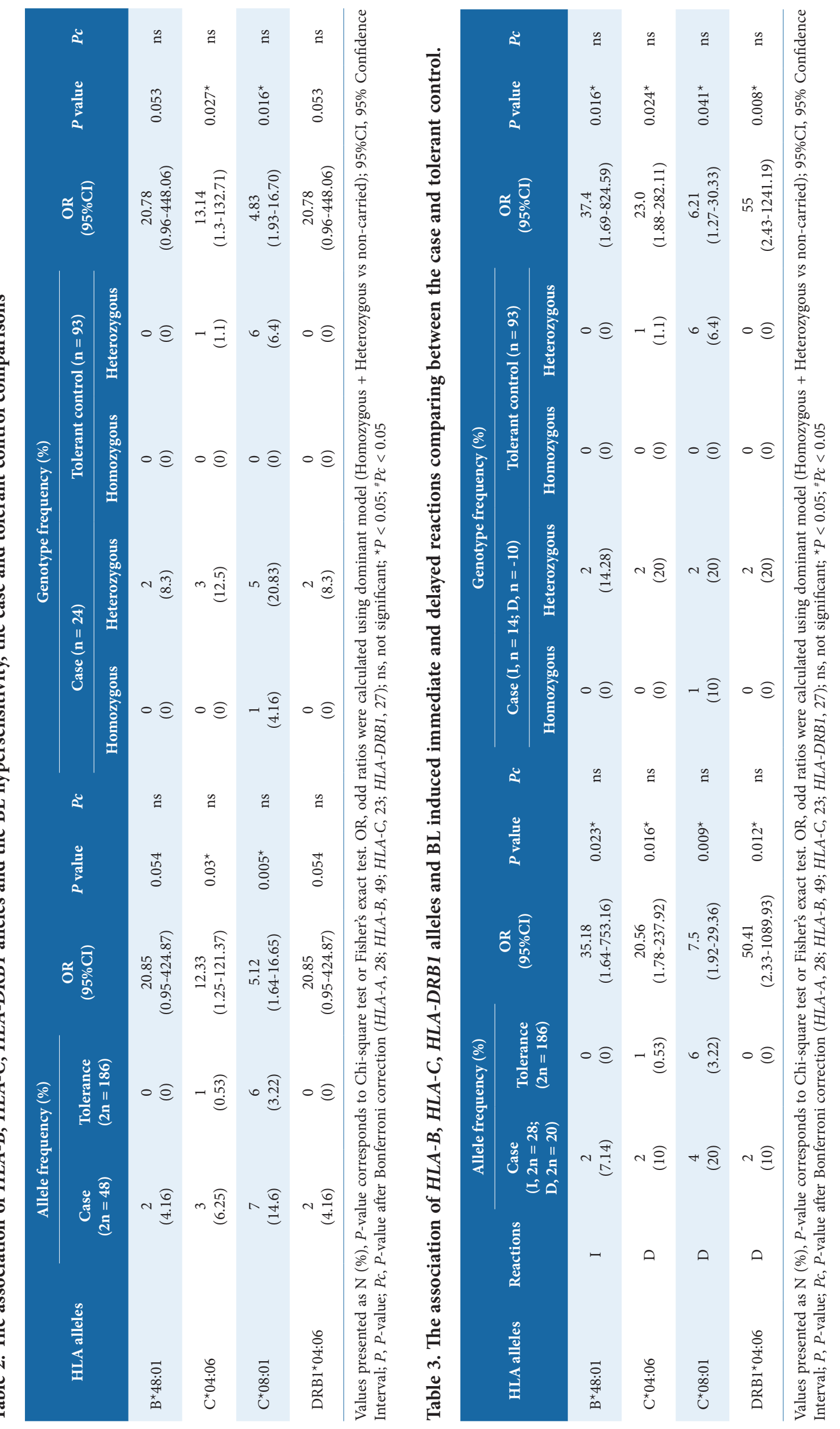

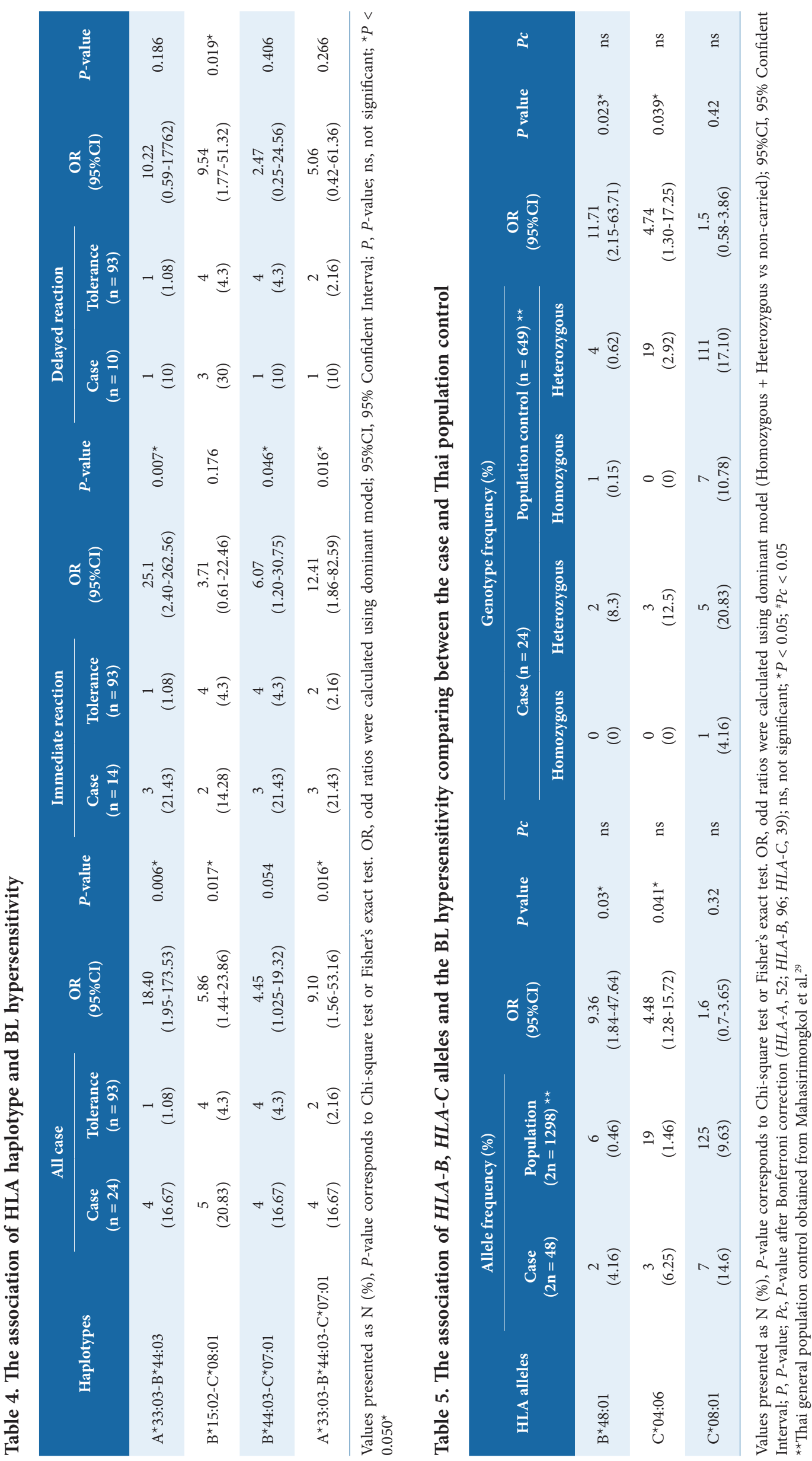


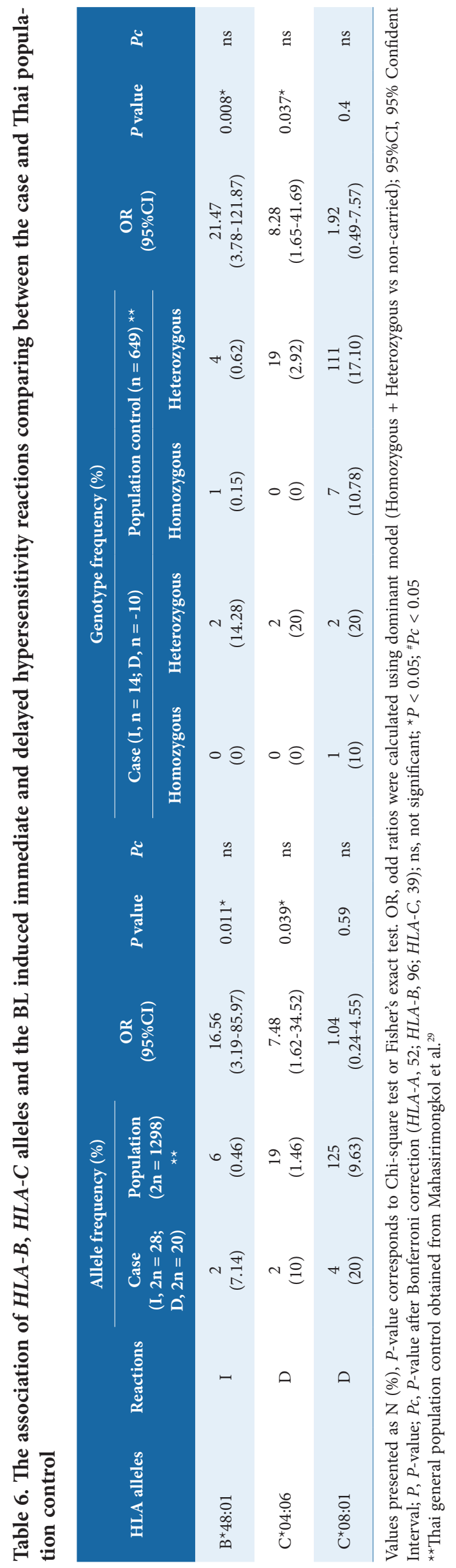


and those who could tolerate $\mathrm{BL}$, respectively $(\mathrm{OR}=13.14$, 95\%CI: $1.3-132.71 ; p=0.027, p c=\mathrm{ns})$. HLA- $C^{\star} 08: 01$ carrier rates were $25 \%$ and $6.4 \%$ in children who had $\mathrm{BL}$ hypersensitivity and those who could tolerate $\mathrm{BL}$, respectively $(\mathrm{OR}=4.83,95 \% \mathrm{CI}: 1.93-16.70 ; p=0.016, p c=\mathrm{ns})$ (Table 2). HLA allele frequency and the OR among those with the $H L A-B^{\star} 48: 01, H L A-C^{\star} 04: 06, H L A-C^{\star} 08: 01, H L A-D R B 1^{\star} 04: 06$ were demonstrated in Table 2 . The haplotype analysis revealed that the frequencies of the $H L A-A^{\star 3} 33: 03-B^{\star} 44: 03$, $H L A-B^{\star} 15: 02-C^{\star} 08: 01$ and $H L A-A^{\star} 33: 03-B^{\star} 44: 03-C^{\star} 07: 01$ haplotypes in the $\mathrm{BL}$ hypersensitivity group were statistically significantly different from those in the tolerant control group. The OR of BL hypersensitivity among patients with the HLA$A^{\star} 33$ :03- $B^{\star} 44$ :03 haplotype was 18.40 (95\%CI, $1.95-173.53$; $p$ $=0.006)$, the OR among those with the HLA- $B^{\star} 15: 02-C^{\star} 08: 01$ haplotype was $5.86(95 \% \mathrm{CI}, 1.44-23.86 ; p=0.017$, and the OR among those with the $H L A-A^{\star} 33: 03-B^{\star} 44$ :03- $C^{\star} 07: 01$ haplotype was 9.10 (95\%CI, 1.56-53.16; $P=0.016$; Table 4).

\section{Association of HLA Allele Carrier Rates and the BL induced immediate or delayed hypersensitivity reaction}

In subgroup analysis according to type of reaction, all children who carried $H L A-B^{\star} 48: 01$ had immediate reaction from BL (OR 37.4; 95\%CI 1.69-824.59; $p=0.016$ ) and all children who carried $H L A-D R B 1^{*} 04: 06$ had delayed reaction from $\mathrm{BL}$ (OR 55; 95\%CI 2.43-1241.19; $p=0.008, p c=n s$ ). Significant association of $H L A-C^{\star} 04: 06$ and $H L A-C^{\star} 08: 01$ with the BL induced delayed hypersensitivity reaction were demonstrated (Table 3). The haplotype analysis revealed that the frequencies of the HLA- $A^{\star} 33: 03-B^{\star} 44: 03, H L A-B^{\star} 44: 03-C^{\star} 07: 01$ and $H L A-$ $A^{\star} 33: 03-B^{\star} 44: 03-C^{\star} 07: 01$ haplotypes in the $\mathrm{BL}$ induced immediate reaction group were statistically significantly different from those in the tolerant control group. The OR of BL hypersensitivity among patients with the $H L A-A{ }^{\star} 33: 03-B{ }^{\star} 44: 03$ haplotype was $25.1(95 \% \mathrm{CI}, 2.40-262.56 ; p=0.007)$, the OR among those with the $H L A-B^{\star} 44: 03-C^{\star} 07: 01$ haplotype was 6.07 (95\%CI, $1.20-30.75 ; p=0.046$, and the OR among those with the $H L A-A^{\star} 33: 03-B^{\star} 44: 03-C^{\star} 07: 01$ haplotype was 12.41 (95\%CI, 1.86-82.59; $p=0.016$; Table 4). Haplotype analysis in $\mathrm{BL}$ induced delayed reaction revealed that only $H L A-$ $B^{\star} 15: 02-C^{\star} 08$ :01 haplotype were statistically significantly different from those in the tolerant control group with the OR 9.54 (95\%CI, 1.77-51.32; $P=0.019)$.

\section{Comparison of HLA allele carrier rates in the BL hypersensi- tivity and in the general Thai population}

Comparison to the HLA allele carrier rate in the general Thai population, $H L A-B^{\star} 48: 01$ and $H L A-C^{\star} 04: 06$ were significantly associated with $\mathrm{BL}$ hypersensitivity $(\mathrm{OR}=11.71$, 95\%CI: 2.15-63.71; $p=0.023, p c=\mathrm{ns}$ and $\mathrm{OR}=4.74,95 \% \mathrm{CI}$ : $1.30-17.25 ; p=0.039, p c=n s$, respectively. However, there was no significant association of $H L A-C^{\star} 08$ :01 with BL hypersensitivity (Table 5). In subgroup analysis according to type of hypersensitivity reaction, $H L A-B^{\star} 48$ :01 was significantly associated with $\mathrm{BL}$ induced immediate hypersensitivity reaction $(\mathrm{OR}=21.47,95 \% \mathrm{CI}: 3.78-121.87 ; p=0.008, p c=\mathrm{ns}$, while $H L A-C^{\star} 04$ :06 was significantly associated with BL induced delayed hypersensitivity reaction $(\mathrm{OR}=8.28,95 \% \mathrm{CI}$ : 1.65-41.69; $p=0.037, p c=$ ns) (Table 6).

\section{Discussion}

We have demonstrated that the prevalence of true $\mathrm{BL}$ hypersensitivity is only $9.8 \%$ in children who had history of suspected of BL hypersensitivity. This finding was similar to a previous study in 783 patients with symptoms suggestive of BL hypersensitivity. Only 62 cases $(7.9 \%)$ were confirmed to have $\mathrm{BL}$ hypersensitivity. ${ }^{19} \mathrm{BL}$ antibiotics are one of the most common drug allergies since they are the most prescribed drugs worldwide. ${ }^{20}$ Investigation for drug hypersensitivity reaction require both skin test and drug provocation test. Among 7 children who were confirmed to have BL hypersensitivity reactions in our study, $3(42.8 \%)$ children were diagnosed by skin tests and $4(57.2 \%)$ by drug provocation test. As a result, both skin test and drug provocation have to be included for drug hypersensitivity investigation.

The current study have shown the associations of $H L A-B^{*}$ 48:01, HLA- $C^{\star} 04: 06, H L A-C^{\star} 08: 01$ and $H L A-D R B 1^{\star} 04: 06$ and BL hypersensitivity. $H L A-B^{\star} 48: 01$ has been shown to be associated with the $\mathrm{BL}$ induced immediate hypersensitivity reactions while $H L A-C^{\star} 04$ :06, $H L A-C^{\star} 08: 01$ and $H L A-D R B 1^{\star} 04: 06$ were shown to be associated with the BL-induced delayed hypersensitivity reactions. Previous studies have demonstrated the associations with Th2 cytokine-related genes and beta-lactam-induced immediate hypersensitivity reactions. ${ }^{21}$ However, there is limited study reporting on the association of beta-lactam-induced immediate reaction and HLA class I genotype. The association with $H L A-D R B 1^{*} 09$ and immediate hypersensitive reaction to penicillin has been reported in Chinese population. ${ }^{22}$ However, we could not demonstrate the association between $H L A-D R B 1^{\star} 09$ and the BL-induced immediate hypersensitivity reaction. There were no children in the current study who developed immediate reaction from $\mathrm{BL}$ who carried $H L A-D R B 1^{\star} 09$. Since the $H L A-B^{\star} 48$ :01 allele frequency in Thai population is only $0.2 \%^{23}$ and the BL-induced immediate hypersensitivity reaction ranging from acute urticaria to severe systemic allergic reaction or anaphylaxis. Based on our result, we would suggest using antibiotic other than beta-lactam antibiotics in Thai children who were known to carry $H L A-B^{\star} 48: 01$ for avoiding the severe immediate drug hypersensitivity reaction.

$H L A-C^{\star} 08: 01$ is a common allele in Thai population with the allele frequency of $15.75 \% .{ }^{24} H L A-C^{\star} 08: 01$ was reported to be associated with co-trimoxazole-induced severe cutaneous reactions in HIV infected Thai adults. ${ }^{24}$ We have shown the association of $H L A-C^{\star} 08: 01$ and the BL-induced delayed hypersensitivity reactions. $H L A-C^{\star} 08: 01$ allele frequency in our tolerant control is $3 \%$, which is significantly lower than Thai general population $(15.75 \%)$, this is the usual patterns of association where the pharmacogenetics risk increase frequency in case and decrease frequency in control groups, when compared with frequency in general population. The low allele frequency of $H L A-C^{\star} 08: 01$ in tolerant control may explain the reason why the significant association of $H L A-C^{*} 08: 01$ and $\mathrm{BL}$ hypersensitivity was demonstrated only in the comparison with tolerant control but not in population control. We hypothesize that $H L A-C^{\star} 08: 01$ possibly be the risk allele for BL hypersensitivity. Since all tolerant control were challenged 
with $\mathrm{BL}$, consequently children who carry at risk allele (such as $\left.H L A-C^{\star} 08: 01\right)$ would develop BL hypersensitivity and converted to cases group, resulting in the low $H L A-C^{*} 08: 01$ allele frequency in our tolerant control. However, a further study with a larger number of children with BL hypersensitivity and tolerant control should be replicated to validate this observation.

We have shown the association of $H L A-C^{\star 04: 06}$ and BL-induced delayed hypersensitivity reactions. Two out of 10 children who had delayed hypersensitivity reaction from $\mathrm{BL}$ carried $H L A-C^{\star} 04: 06$ while only 1 in 93 tolerant control children carried this allele. However, there is no previous report on the association of $H L A-C^{\star} 04: 06$ and drug hypersensitivity. $H L A-C^{\star} 04: 06$ is an uncommon HLA allele in Thais and worldwide, $H L A-C^{\star} 04: 06$ allele frequency was reported as low as 0 to $0.82 \%$. $^{25}$

The allele frequencies of $H L A-D R B 1^{\star} 04$ :06 in Thai population were reported ranging from $0.2-4.3 \%{ }^{25}$ A recent study has reported the association of $H L A-D R B 1^{\star} 04$ :06 in statin-related myopathy in Japanese patients. ${ }^{26}$ However, there is no previous report on the role of $H L A-D R B 1^{*} 04: 06$ and BL hypersensitivity reactions. We have demonstrated the associations of $H L A-C^{\star} 04: 06, H L A-C^{\star} 08: 01$ and $H L A-D R B 1^{*} 04: 06$ and the $\mathrm{BL}$ induced delayed hypersensitivity reactions. $\mathrm{BL}$ induced delayed hypersensitivity reactions is the $\mathrm{T}$ cell mediated drug hypersensitivity requiring T cell-HLA interaction. A recent study have demonstrated the shared peptide binding of HLA Class I and II alleles associate with cutaneous nevirapine hypersensitivity. ${ }^{27}$ However, we did not evaluate for the similarities between binding specificities for our identified HLA risk alleles.

The current study have shown that the frequencies of the $H L A-A^{\star} 33: 03-B^{\star} 44: 03, H L A-B^{\star} 15: 02-C^{\star} 08: 01$ and $H L A-$ $A^{\star} 33: 03-B^{\star} 44: 03-C^{\star} 07: 01$ haplotypes in the BL hypersensitivity group were statistically significantly different from those in the tolerant control group. $H L A-A^{\star} 33: 03-B^{\star} 44: 03$ and $H L A$ $B^{\star} 15: 02-C^{\star} 08$ :01 haplotype were reported as haplotype risk alleles for cold medicine-related Stevens-Johnson syndrome with severe ocular complication ${ }^{28}$ and for co-trimoxazole-induced severe cutaneous reaction ${ }^{24}$ in Thailand. Since BL is commonly used for the treatment of respiratory tract infections. It is high possibility that our enrolled patients had been prescribed with other cold medicines along with BL resulting the association of these haplotypes and BL hypersensitivity in the current study.

The strength of our study is that all of BL hypersensitivity cases except for BL induced SCARs underwent investigation for drug hypersensitivity including skin test and drug provocation test and all of the control cases were BL tolerant control. However, our study still has limitations. Firstly, we have enrolled a limited number of SCARs. Secondly, there were no BL tolerant control children carried $H L A-B^{\star} 48: 01$, and $H L A-D R B 1^{\star} 04: 06$ alleles due to these HLA alleles are uncommon in Thais which may have an effect on the wide range of 95\%CI for these two alleles. A larger sample size in both case and tolerant control may be needed to validate our result.
In conclusion, we have shown that true BL hypersensitivity is approximately $10 \%$ in children with history of BL hypersensitivity. $H L A-B^{\star} 48: 01, H L A-C^{\star} 04: 06, H L A-C^{\star} 08: 01$ and $H L A-D R B 1^{*} 04: 06$ may be associated with the BL hypersensitivity in Thai children. These findings highlight the potential role for HLA genotype in predicting the BL hypersensitivity in children.

\section{Acknowledgement}

We would like to thank Professor John Carey for helping in editing the manuscript. This study was financially supported by National Research Council of Thailand under the project entitled "Pharmacogenomics studies of common adverse reactions in Thais (PGx-COMART)" to Dr Surakameth Mahasirimongkol and Department of Medical Sciences, Ministry of Public Health, Nonthaburi, Thailand.

\section{Disclosure of conflicts of interest}

None of the authors have any conflicts of interest to disclose.

\section{References}

1. Solensky R. Allergy to beta-lactam antibiotics. J Allergy Clin Immunol. 2012;130:1442-5.

2. Manuyakorn W, Singvijarn P, Benjaponpitak S, Kamchaisatian W, Rerkpattanapipat T, Sasisakulporn C, et al. Skin testing with beta-lactam antibiotics for diagnosis of beta-lactam hypersensitivity in children. Asian Pac J Allergy Immunol. 2016;34:242-7.

3. Smyth RM, Gargon E, Kirkham J, Cresswell L, Golder S, Smyth R, et al. Adverse drug reactions in children--a systematic review. PLoS One. 2012; 7:e24061.

4. Romano A. Recognising antibacterial hypersensitivity in children. Paediatr Drugs. 2000;2:101-12.

5. Romano A, Blanca M, Torres MJ, Bircher A, Aberer W, Brockow K, et al Diagnosis of nonimmediate reactions to beta-lactam antibiotics. Allergy. 2004;59:1153-60

6. Wohrl S, Vigl K, Stingl G. Patients with drug reactions -- is it worth testing? Allergy. 2006;61:928-34.

7. Maagdenberg $\mathrm{H}$, Vijverberg SJH, Bierings $\mathrm{MB}$, Carleton BC, Arets HGM, de Boer A, et al. Pharmacogenomics in Pediatric Patients Towards Personalized Medicine. Pediatric Drugs. 2016;18:251-60.

8. Cheung YK, Cheng SH, Chan EJ, Lo SV, Ng MH, Kwan P. HLA-B alleles associated with severe cutaneous reactions to antiepileptic drugs in Han Chinese. Epilepsia. 2013;54:1307-14.

9. Tassaneeyakul W, Tiamkao S, Jantararoungtong T, Chen P, Lin SY, Chen WH, et al. Association between HLA-B ${ }^{\star} 1502$ and carbamazepine-induced severe cutaneous adverse drug reactions in a Thai population. Epilepsia. 2010;51:926-30.

10. Locharernkul C, Loplumlert J, Limotai C, Korkij W, Desudchit T, Tongkobpetch S, et al. Carbamazepine and phenytoin induced Stevens -Johnson syndrome is associated with HLA-B ${ }^{\star} 1502$ allele in Thai population. Epilepsia. 2008;49:2087-91.

11. McCormack M, Alfirevic A, Bourgeois S, Farrell JJ, Kasperaviciute $\mathrm{D}$, Carrington $\mathrm{M}$, et al. HLA-A*3101 and carbamazepine-induced hypersensitivity reactions in Europeans. N Engl J Med. 2011;364:1134-43.

12. Amstutz U, Shear NH, Rieder MJ, Hwang S, Fung V, Nakamura H, et al. Recommendations for HLA-B ${ }^{\star}$ 15:02 and HLA-A ${ }^{\star} 31: 01$ genetic testing to reduce the risk of carbamazepine-induced hypersensitivity reactions. Epilepsia. 2014;55:496-506.

13. Caudle KE, Rettie AE, Whirl-Carrillo M, Smith LH, Mintzer S, Lee MT, et al. Clinical pharmacogenetics implementation consortium guidelines for CYP2C9 and HLA-B genotypes and phenytoin dosing. Clin Pharmacol Ther. 2014;96:542-8.

14. Suvichapanich S, Jittikoon J, Wichukchinda N, Kamchaisatian W, Visudtibhan A, Benjapopitak S, et al. Association analysis of CYP2C9*3 and phenytoin-induced severe cutaneous adverse reactions (SCARs) in Thai epilepsy children. J Hum Genet. 2015;60:413-7. 
15. Manuyakorn W, Siripool K, Kamchaisatian W, Pakakasama S, Visudtibhan A, Vilaiyuk S, et al. Phenobarbital-induced severe cutaneous adverse drug reactions are associated with CYP2C19*2 in Thai children. Pediatr Allergy Immunol. 2013;24:299-303.

16. Manuyakorn W, Mahasirimongkol S, Likkasittipan P, Kamchaisatian W, Wattanapokayakit S, Inunchot W, et al. Association of HLA genotypes with phenobarbital hypersensitivity in children. Epilepsia. 2016;57:1610-6.

17. Roujeau JC, Stern RS. Severe adverse cutaneous reactions to drugs. N Engl J Med. 1994;331:1272-85.

18. Kardaun SH, Sidoroff A, Valeyrie-Allanore L, Halevy S, Davidovici BB, Mockenhaupt $M$, et al. Variability in the clinical pattern of cutaneous side-effects of drugs with systemic symptoms: does a DRESS syndrome really exist? Br J Dermatol. 2007;156:609-11.

19. Zambonino MA, Corzo JL, Munoz C, Requena G, Ariza A, Mayorga C, et al. Diagnostic evaluation of hypersensitivity reactions to beta-lactam antibiotics in a large population of children. Pediatr Allergy Immunol. 2014;25:80-7.

20. Ponvert C, Perrin Y, Bados-Albiero A, Le Bourgeois M, Karila C, Delacourt C, et al. Allergy to betalactam antibiotics in children: results of a 20 -year study based on clinical history, skin and challenge tests. Pediatr Allergy Immunol. 2011;22:411-8.

21. Khan DA. Pharmacogenomics and adverse drug reactions: Primetime and not ready for primetime tests. J Allergy Clin Immunol. 2016;138:943-55.

22. Yang J, Qiao HL, Zhang YW, Jia LJ, Tian X, Gao. HLA-DRB genotype and specific IgE responses in patients with allergies to penicillins. Chin Med J (Engl). 2006;119:458-66.
23. Mahasirimongkol S, Kumperasart S, Wattanapokayakit S, Satproedprai N, Inunchot W, Aekplakorn W, et al. HLA-B Allele Distribution in Samples from Thailand National Health Examination Survey. J Health Sci. 2014; 23:191-200.

24. Kongpan T, Mahasirimongkol S, Konyoung P, Kanjanawart S, Chumworathayi P, Wichukchinda N, et al. Candidate HLA genes for prediction of co-trimoxazole-induced severe cutaneous reactions. Pharmacogenet Genomics. 2015;25:402-11.

25. Gonzalez-Galarza FF, Takeshita LY, Santos EJ, Kempson F, Maia MH, Silva AL, et al. Allele frequency net 2015 update: new features for HLA epitopes, KIR and disease and HLA adverse drug reaction associations. Nucleic Acid Res. 2015;43:D784-8.

26. Sai K, Kajinami K, Akao H, Iwadare M, Sato-Ishida R, Kawai Y, et al. A possible role for HLA-DRB1 ${ }^{\star}$ 04:06 in statin-related myopathy in Japanese patients. Drug Metab Pharmacokinet. 2016;31:467-70.

27. Pavlos R, McKinnon EJ, Ostrov DA, Peters B, Buus S, Koelle D, et al. Shared peptide binding of HLA Class I and II alleles associate with cutaneous nevirapine hypersensitivity and identify novel risk alleles. Sci Rep. 2017;7:8653.

28. Jongkhajornpong $\mathrm{P}$, Lekhanont $\mathrm{K}$, Pisuchpen $\mathrm{P}$, Chantaren $\mathrm{P}$, Puangsricharern V, Prabhasawat $\mathrm{P}$, et al.Association between HLA-B ${ }^{\star} 44$ : 03-HLA-C ${ }^{\star}$ 07:01 haplotype and cold medicine-related Stevens-Johnson syndrome with severe ocular complications in Thailand. Br J Ophthalmol. 2018;102:1303-7.

29. Mahasirimongkol S, Somboonyosdech C, Kumperasart S, Wattanapokayakitet S, Satproedprai N, Inunchot W, et al. HLA-B allele distribution in samples from Thailand National Health Examination Survey. J Health Sci. 2014;23:191-200. 